\title{
Simulation of Optical Properties of Inhomogeneous Molecular Contamination Thin Film Outgassed from Non-Metallic Materials
}

\author{
Jia QIAO*
}

\begin{abstract}
Molecular contamination outgassing from non-metallic spacecraft materials is deposited on sensitive surfaces after diffusion, thereby degrading the performance of the optical device. To investigate the effect of nonmetal contamination on optics, non-metallic mixing film was prepared on a glass substrate using an improved method of ASTM E 1559, and the transmittance was measured using a spectrophotometer. To more accurately simulate the transmittance of contamination film, an optical contamination effect model was established based on diffusion theory, angle coefficient method, first-order desorption equation, Lorentz-Lorenz relation and wave propagation in hierarchical media and dielectric film theory. The simulation results are in good agreement with the experimental values.
\end{abstract}

Keywords: dielectric film theory; Lorentz-Lorenz relation; optical contamination effect model; wave propagation in hierarchical media

\section{INTRODUCTION}

Molecular contamination outgassing from nonmetallic spacecraft materials is deposited on the sensitive surfaces after diffusion under vacuum and high temperature. The contamination molecules deposited on the surface of solar cells decrease the output power of the solar cells. Moreover, those deposited on optical surfaces affect the transmittance and reflectivity of the optical devices. When silicone oil films deposited on the highresolution optical facilities of satellites are as thick as several tens of nanometers, the reflectance of light with a wavelength of $0.13 \mu \mathrm{m}$ decreases by $10 \%$ [1-3].

Extensive studies on the effect of contamination on optical devices have been conducted. Yong et al. [4, 5] studied the optical properties of hydrogen plasma-treated aluminum-doped $\mathrm{ZnO}$ thin films. They found that the resistivity of the plasma-treated samples decreases, the refractive index decreases in the wavelength range of 350$1100 \mathrm{~nm}$, and the extinction coefficient decreases in a short wavelength range and remains unchanged in the longwavelength range owing to the improvement in the number of oxygen vacancies and $\mathrm{O}-\mathrm{H}$ bonds. Hossain et al. [6, 7] studied the influence of the thickness of gamma-irradiated photoanodic $\mathrm{TiO}_{2}$ film on its structural, morphological, and optical properties. The results show that the optical bandgap of the film increases with an increase in the film thickness. Masoud et al. [8, 9] evaluated the optical properties of heterostructured $\mathrm{NiO}-\mathrm{TiO} 2$ film used for photovoltaic (PV) modules, and they found that the film increases the absorption coefficient of the solar absorber plates up to $98 \%$ in the ultraviolet (UV) range. In the infrared region, the absorption coefficient for dispersion was decreased to $2 \%$. The above-cited studies reported only the factors affecting the optical properties of thin films but did not give a specific prediction model. Bertrand et al. [10] used the Lorentz-Lorenz relationship to model the deposit on the optical surface of a spacecraft containing a mixture of molecular species. However, the concentration of the absorbing molecules and the degree of hydrogen bonding of water molecules may be in error. The measured spectrum is greater than the calculated one. In 2018, the Japan Aerospace Exploration Agency [10] studied the effect of contamination films on optical performance during the outgassing and desorption of epoxy resin based on HOM [11] and EMA [12,13] and compared the calculated contaminated thickness with the actual one to validate the established model. Furthermore, they employed a least-square to obtain 11 parameters using the model. According to [19], the dielectric constant changes significantly in the range of $10 \mathrm{~nm}$, remains almost unchanged above this range. Ref. [10] did not consider this range, leading to an error between the calculated and experimental values.

Optical effect models for inhomogeneous molecular contamination films have been extensively studied. Herein, we developed optical contamination effects models for thin films based on wave propagation in hierarchical media and dielectric film theory. The Goos-Male [16] model was used to compute the optical constants of layers produced by every material. The Lorentz-Lorenz relationship was used to obtain the optical constants of rough surfaces and mixing layers produced by several materials. Then, the outgassing deposition process was dynamically simulated, and the transmittance of contamination films was calculated by incorporating the optical constants and simulation results of the outgassing deposition process into the wave optics module of COMSOL. Finally, we conducted an experiment using RTV and Uralane as contamination sources, which were deposited on the surface of optical glass substrates, to study the transmittance of the contaminated optical glass in the IR region. The experimental and simulation results were compared to verify the feasibility of the model.

\section{ESTABLISHMENT OF MODEL}

The angular coefficient method and wave optics module of COMSOL were employed to simulate the outgassing deposition process and optical effect of molecular contamination.

\subsection{Contamination Model}

To accurately simulate the test process, a geometric model based on ASTM E 1559 was developed [20], which comprises a vacuum test chamber, an outgassing unit, a vacuum pump, and three quartz crystal microbalance (QCM) detectors with the same view factor. The vacuum 
cabin is a cylinder of length $360 \mathrm{~mm}$ and radius $200 \mathrm{~mm}$. The outgassing unit is a cylinder with an inner diameter of $65 \mathrm{~mm}$ and a depth of $50 \mathrm{~mm}$. This unit has a cylindrical outgassing hole with an inner diameter equal to its depth (3 $\mathrm{mm})$. The tubular vacuum pump (inner diameter $=140 \mathrm{~mm}$ ) operates at $500 \mathrm{l} / \mathrm{s}[23]$.

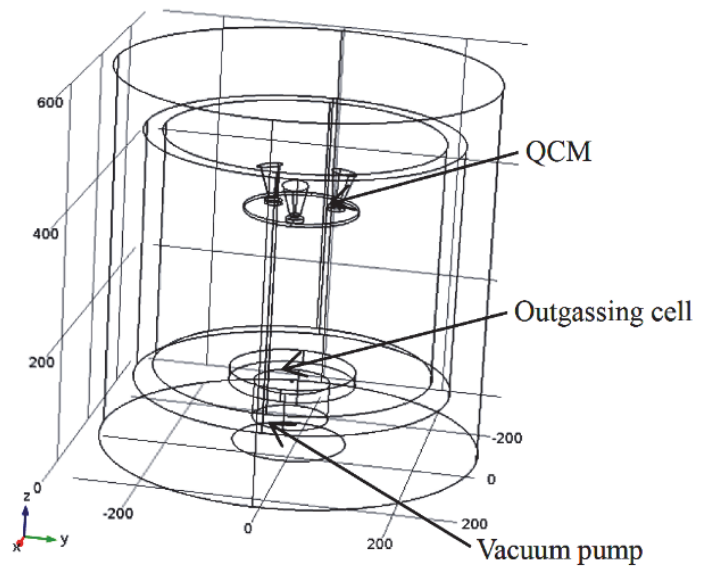

Figure 1 Computational model for experiment equipment

\subsubsection{Outgassing Boundary Condition}

The outgassing orifice was set as the outgassing boundary in the outgassing model, which is based on diffusion theory [21-23]:

$$
m=0.5 C_{0} d\left[1-\frac{8}{\pi^{2}} \exp \left(\frac{\pi^{2} D t}{d^{2}}\right)\right]
$$

where $m$ is outgassing mass, $C_{0}$ the initial concentration, $d$ the thickness of sample, and $D$ the diffusion coefficient. $C_{0}$ and $D$ are obtained by experiment, and the outgassing rate is as follows:

$$
\frac{\mathrm{d} m}{\mathrm{~d} t}=\frac{4 C_{0} D}{d} \exp \left(\frac{\pi^{2} D t}{d^{2}}\right)
$$

\subsubsection{Deposition Boundary Condition}

In the experimental setup, the chamber wall is cooled by liquid nitrogen; thus, it attracts most of the contaminant molecules. Therefore, this wall was set as the adsorption wall with a sticking coefficient of 1.0. The surfaces of the three QCMs were set as the adsorption/desorption walls. In COMSOL, the simulated desorption and adsorption of molecules are controlled by the molar desorption rate $\left(D_{1}\right)$ and a sticking coefficient $(S)$ :

$J=(1-S) G+D_{1} N_{\mathrm{A}}$

$\frac{\mathrm{d} n_{\mathrm{ads}}}{\mathrm{d} t}=\frac{S G}{N_{\mathrm{A}}}-D_{1}+\Gamma$

where $G$ is the incident molecular flux, $J$ the emitted molecular flux, $N_{\mathrm{A}}$ the Avogadro's number, $n_{\mathrm{ads}}$ the molar concentration of adsorbed molecules, $\Gamma$ an additional surface source of molar flux.
In this model the sticking coefficient $S$ is given as

$S=S_{0}\left(1-\frac{n_{\text {ads }}}{n_{\text {sites }}}\right)$

where $S_{0}$ is sticking coefficient of a clean surface, and is assumed to be $1, n_{\text {sites }}$ is the molar capacity of the surface sites on the system (assumed to be $1 \times 10^{-4} \mathrm{~mol} / \mathrm{m}^{2}$ ) $[13,14]$. The desorption rate is expressed as follows:

$D=\frac{n_{\text {ads }}}{\tau}$

where $\tau$ is the residence time. Eq. (6) assumes that the desorption process is first order

$\tau=\tau_{0} \exp \left(\frac{E_{\mathrm{a}}}{R T}\right)$

where $E_{\mathrm{a}}$ is the chemical energy of desorption, $\tau_{0}$ the lattice vibration time (assumed to be $10^{-13} \mathrm{~s}$ ) [13-15, 23].

\subsection{Optical Effect Model of Contamination Films}

Herein, films formed by molecular contamination outgassing from two nonmetallic materials deposited on the optical surface were investigated and the rough surface

\begin{tabular}{|c|c|}
\hline$n_{\text {surf }} k_{\text {surf }} d_{\text {surf }}$ & Surface layer \\
\hline & Mixing layer \\
\hline $\mathrm{n}_{\mathrm{s}}$ & substrate \\
\hline
\end{tabular}
was considered. The geometric model is shown in Fig. 2.

Figure 2 Schematic diagram of the Optical contamination effect model

Therefore, the main influencing parameters of the optical contamination effect model include the film thickness $\left(d_{j}\right)$ of each layer, the refractive index $n_{j}$, the extinction coefficient $k_{j}$ of each layer, and the number of layers, the film thickness can be acquired by the contamination simulation.

In the multilayer model [16], if the feature matrix of each film layer is given $\left[\begin{array}{cc}\cos \delta_{j} & \frac{i}{\eta_{j}} \sin \delta_{j} \\ i \eta_{j} \sin \delta_{j} & \cos \delta_{j}\end{array}\right]$, and the spectral transmittance value of the multilayer can be obtained as follows:

$\left[\begin{array}{l}B \\ C\end{array}\right]=\left\{\prod_{j=1}^{K}\left[\begin{array}{cc}\cos \delta_{j} & \frac{i}{\eta_{j}} \sin \delta_{j} \\ i \eta_{j} \sin \delta_{j} & \cos \delta_{j}\end{array}\right]\right\}\left[\begin{array}{c}1 \\ \eta_{K+1}\end{array}\right]$ 
where $\eta_{j}$ and $\delta_{j}$ are related to the refractive index $n_{j}$, the extinction coefficient $k_{j}$, the incident angle $\theta_{j}$ and the film thickness $d_{j}$ of layer $j$.

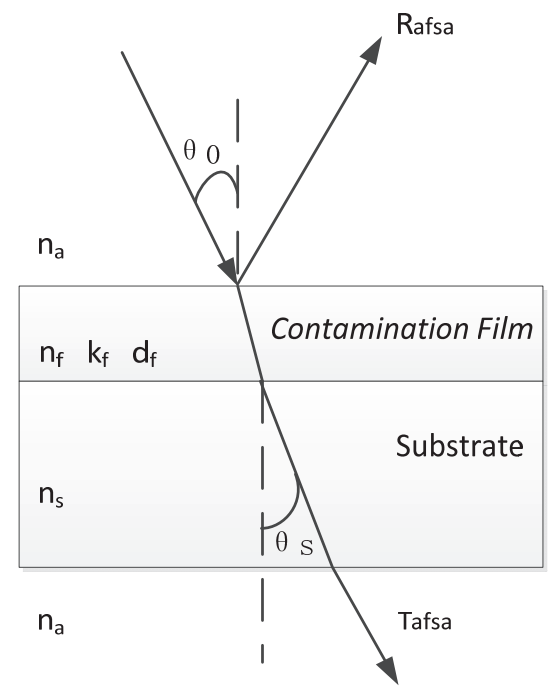

(a)

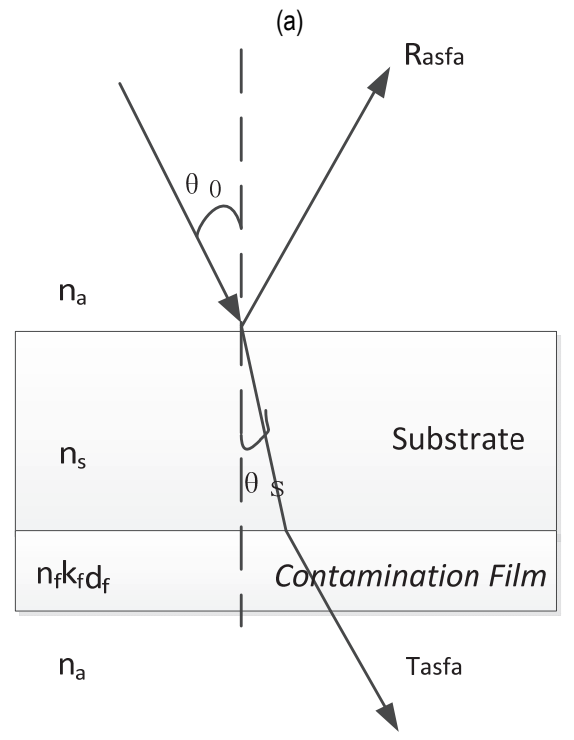

(b)

Figure 3 (a) Light illumination of the surface of a contamination film (b) Light illumination of the surface of the substrate

Potential transmission:

$R=\left(\frac{\eta_{0} B-C}{\eta_{0} B+C}\right)\left(\frac{\eta_{0} B-C}{\eta_{0} B+C}\right)^{*}$

$$
T=\frac{4 \eta_{0} \operatorname{Re}\left(\eta_{K+1}\right)}{\left(\eta_{0} B+C\right)\left(\eta_{0} B+C\right)^{*}}
$$

From this, the optical constants, $n_{j}, k_{j}, \theta_{j}$ and $d_{j}$, can be measured to obtain the transmittance of the contaminated film.

\subsection{Acquisition Method of Optical Constants 2.3.1 Calculation of Optical Constants of the Film Layer Produced by a Single Material}

The Goos-Male method was used to calculate the optical constants of the film layer produced by a single material. Fig. 3 shows a schematic of the experimental measurement. An $s$-polarized light with a wavelength of $\lambda$ is used to firstly illuminate the sample surface at an incident angle of $\theta_{0}$ to measure the reflected and transmitted light $R_{\mathrm{afsa}}^{s}$ and $T_{\mathrm{afsa}}^{s}$, respectively (the calculation method for optical constants of p-polarized light is the same with this one), as shown in Fig. 3a, then the light is irradiated to the substrate from the back to measure the reflected and transmitted light $R_{\text {asfa }}^{s}$ and $T_{\text {asfa }}^{s}$, respectively, where the subscripts represent the sequence of the layers, $a$ denotes air, $f$ indicates a contaminated film, and $s$ indicates a substrate. If the test equipment is normal, $T_{\mathrm{afsa}}^{s}=T_{\mathrm{asfa}}^{s}$, and finally, it is expressed to calculate the optical constants of the thin films and the contaminated film thickness [17].

\subsubsection{Calculation of Optical Constants of Mixing Layer Produced by two Materials and Rough Surface}

The Lorentz-Lorenz relation can relate the number density $(N)$ of each component in the material to the optical constants $(\tilde{n}=n+i k)$ [10]. For a single material, the Lorentz-Lorenz relation between the optical constants and the mass density $(\rho=m N)$ is as follows:

$$
\frac{\rho^{-1}\left(\tilde{n}^{2}-1\right)}{\left(\tilde{n}^{2}+b\right)}=\frac{a \alpha}{m}
$$

where $m$ is the average mass of the molecule, $\alpha$ the average polarizability of every molecule, $b$ a local parameter, and $a$ another local electric field parameter.

If the uniform dielectric constant contains only nonpolarized molecules, then

$$
a=\left(3 \varepsilon_{0}\right)^{-1}, b=2
$$

The Lorentz-Lorenz relationship was used to calculate the optical constants of the mixing layer according to those of every constituent. The optical constants of the rough surface were also calculated according to those of the mixing layer and the air. The Lorentz-Lorenz relationship can relate the optical constants for the $j$-th pure constituent material to the polarizability of the molecules in the pure $j$ th material as follows [19]:

$$
\alpha=\frac{\left(N_{\mathrm{p} j} a_{j}\right)^{-1}\left(\tilde{n}_{j}^{2}-1\right)}{\left(\tilde{n}_{j}^{2}+b_{j}\right)}
$$

$N_{\mathrm{p} j}$ is the number density of the $j$-th constituent in the pure material, $a_{j}$ and $b_{j}$ are the appropriate local field parameter of the pure material, the average molecular polarizability of the mixture $\alpha$ is the average mass of the polarizability of each constituent in the mixture, expressed as follows:

$$
\alpha=\frac{\Sigma N_{\mathrm{pj}} a_{j}}{N}
$$


Combining Eqs. (11), (12), and (14), the optical constants in the mixture can be expressed as follows:

$$
\begin{aligned}
& \tilde{n}=[(1+b A) /(1-A)]^{1 / 2} \\
& A=\left[\rho /(1+b) \Sigma\left[\left(m_{j} / m\right)\left(1+b_{j}\right) / \rho_{\mathrm{p} j}\right]\left(\tilde{n}_{j}^{2}-1\right) /\left(\tilde{n}_{j}^{2}+b_{j}\right)\right.
\end{aligned}
$$

$\rho$ is the density of the mixture, $\rho_{\mathrm{p} j}$ the density of the pure material, $m_{j}$ the average mass of the molecules of the pure material.

Take $b=b_{j}=2$, and assume that there are only two materials, and each is approximately pure, then

$$
A=\frac{\rho m_{1}\left(\tilde{n}_{1}^{2}-1\right)}{m \rho_{p 1}\left(\tilde{n}_{1}^{2}+2\right)}+\frac{\rho m_{2}\left(\tilde{n}_{2}^{2}-1\right)}{m \rho_{p 2}\left(\tilde{n}_{2}^{2}+2\right)}
$$

where $\rho, m, \rho_{\mathrm{p} 1}, m_{1}, \rho_{\mathrm{p} 2}, m_{2}$ can be acquired by measurement.

Therefore, the optical constants of the rough surface and mixing layer can be obtained by combining combing Eqs. (17) and (15).

\section{EXPERIMENTAL}

A ground test was conducted to investigate the effects of outgassing contamination of nonmetallic materials on the transmittance and reflectance of optical components. First, an outgassing test based on ASTM E1559 was conducted. Second, the transmittance and reflectance of the optical components were measured using a spectrophotometer.

The test process is as follows: The as-prepared RTV and Uralane were cut into pieces and weighed. The sample was heated to $398 \mathrm{~K}$ under a vacuum of $10^{-4} \mathrm{~Pa}$, and the $\mathrm{Ge}$ glass temperature was maintained at $90 \mathrm{~K}$ for $24 \mathrm{~h}$. Singlelayer contaminated films on $\mathrm{Ge}$ glass substrates were obtained. Mixing contaminated layers with two materials were obtained following the above process. The reflectance and transmittance of the deposited glass were measured using a spectrophotometer.

\section{RESULTS AND DISCUSSION}

We calculated the optical transmittance of films composed of several materials based on the optical constants and thickness of every layer film. This was achieved in two parts:

(1) First, parameters $C_{0}$ and $D$ and optical constants of each material were calculated.

$C_{0}$ and $D$ were obtained using an apparatus for measuring the effect of outgassing contamination condensed on a cryogenic sensitive surface in space [18]. The test materials were Uralane and RTV silicone rubbers. The temperatures of the outgassing and deposition plate were 398 and $90 \mathrm{~K}$, respectively. During the parameter test, the sample was heated evenly in a vacuum environment and removed after 2 and $4 \mathrm{~h}$ for weighing. The change in the mass of the sample after removal was divided by the heating time to obtain the average outgassing rate of the material in 1 and $2 \mathrm{~h}$, respectively. The results are shown in Tab. 1.

Table 1 Model parameters
\begin{tabular}{|c|c|c|}
\hline Material & $C_{0} / \mathrm{kg} / \mathrm{m}^{3}$ & $D / \mathrm{m}^{2} / \mathrm{min}$ \\
\hline RTV & 8.2 & $2.34 \times 10^{-10}$ \\
\hline Uralane & 10 & $3.34 \times 10^{-10}$ \\
\hline
\end{tabular}

The single-layer film optical constant calculation program in section 2.3.1 was used to calculate $n(\lambda)$ and $k(\lambda)$ values based on the spectral transmittance and reflectance of layer produced by a single material. On the other hand, the mixing-layer and rough-surface optical-constant models in section 2.3.2 were used to calculate $n(\lambda)$ and $k(\lambda)$ values for the mixing layer and rough surface based on the optical constants of a layer produced by a single material.

(2) Second, the known conditions were incorporated into the wave optics module of COMSOL.

The corresponding transmittance of the multi-layer film at every wavelength $\lambda$ could be calculated by incorporating $n_{j}(\lambda), k_{j}(\lambda), d_{j}$ and the incident angle $\theta_{0}$ into the optical contamination effect model for multilayer films. The transmittance of the multilayer contaminated film was compared with the measured value to verify the accuracy of the model and calculation results.

\subsection{Calculation of Optical Constants of the Layer Produced by Single Material}

The optical constant calculation model for layers produced by a single material was used to calculate $n(\lambda)$ and $k(\lambda)$ values based on the spectral transmittance and reflectance of outgassing contaminated film of Uralane or RTV silicone rubbers deposited on the Ge glass substrate. The calculated $n_{1}, k_{1}, n_{2}, k_{2}$ are shown in Figs. 4 to 7. Fig. 8 shows the calculated thickness of contamination depositing on QCM. The models for the optical constant of the mixing layer and rough surface were used to calculate $n_{\mathrm{t}}, k_{\mathrm{t}}$ for the mixing layer and $n_{\text {surf }}, k_{\text {surf }}$ for the rough surface as shown in Figs. 9 to 12. Fig. 13 shows the calculated and measured value of the transmittance for $0.89 \mu \mathrm{m}$-thick contaminated film.

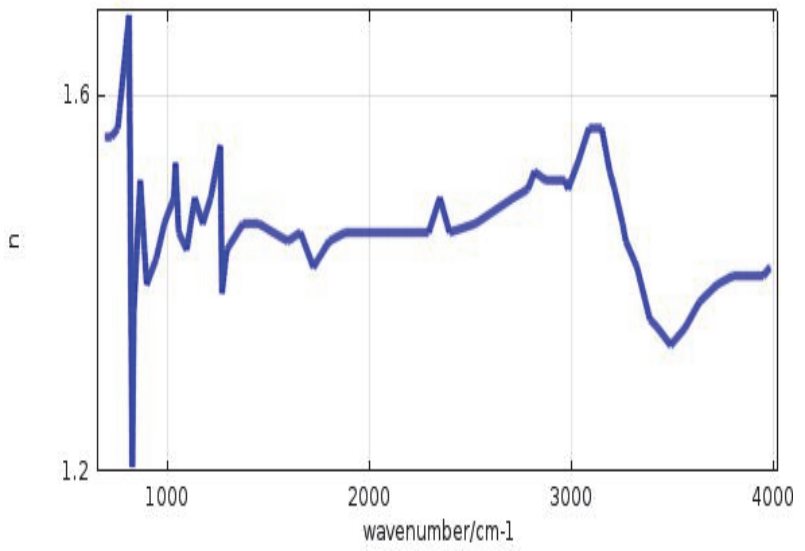

Figure 4 Calculated refractive index $n_{1}$ value of contamination film from the RTV deposited on Ge substrate

The calculated refractive index $n_{1}$ of RTV remains between 1.4 and 1.5 in the band of $1300-2400 \mathrm{~cm}^{-1}$. At $1000 \mathrm{~cm}^{-1}, 1250 \mathrm{~cm}^{-1}, 3100 \mathrm{~cm}^{-1}$, the corresponding $n_{1}$ value shows peak because of the absorption peak of the transmittance of RTV. $k_{1}$ fluctuates rapidly with a change in wavelength, varying from $10^{-4}$ to 0.3 , it increases sharply, particularly at $1000 \mathrm{~cm}^{-1}, 1250 \mathrm{~cm}^{-1}, 3100 \mathrm{~cm}^{-1}$ 
and so on, where the transmittance and reflectivity have absorption peaks.

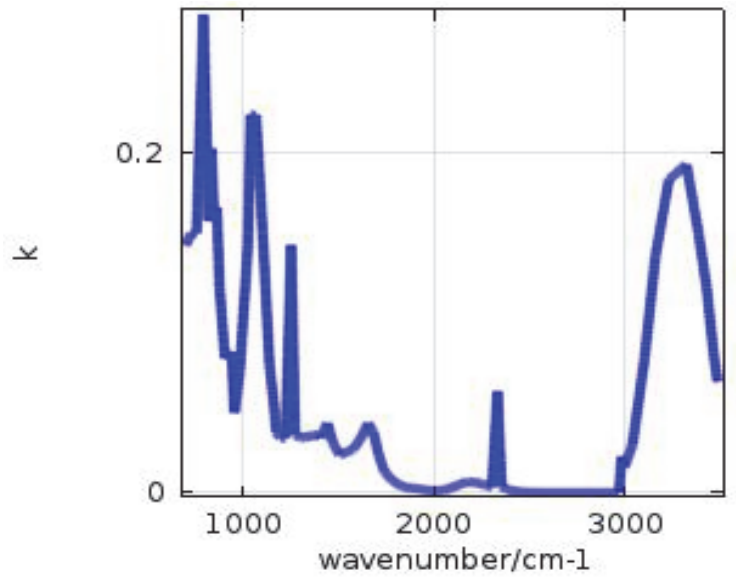

Figure 5 Calculated extinction coefficient $k_{1}$ value of contamination film from RTV deposited on Ge substrate

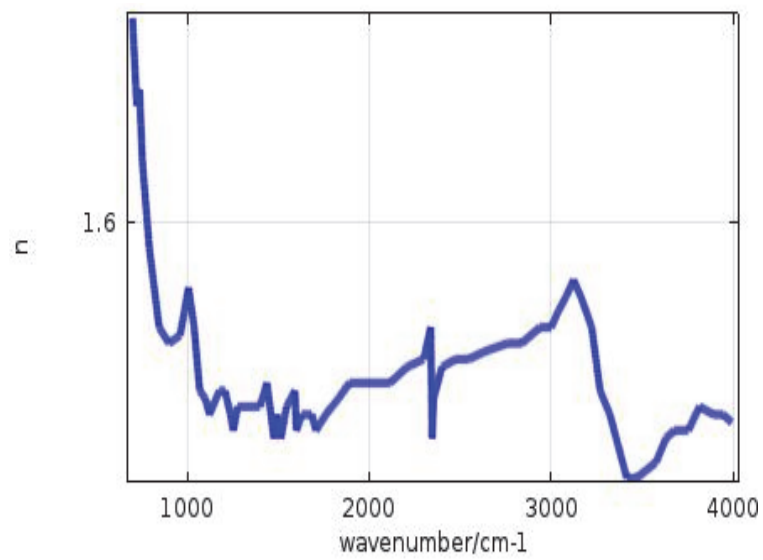

Figure 6 Calculated refractive index $n_{2}$ value of contamination film from the Uralane deposited on Ge substrate

The calculated refractive index $n_{2}$ of the molecular contamination outgassing from Uralane is basically around 1.4 in the infrared band $\left(1,000-4,000 \mathrm{~cm}^{-1}\right)$. The extinction coefficient $k_{2}$ fluctuates rapidly with a change in wavelength, varying from $10^{-4}$ to 0.3 .

Figs. 4 to 7 show the calculated refractive index and extinction coefficients of films obtained from RTV and Uralane. The thickness of the contamination film produced by Uralane and RTV silicone rubbers is shown in Figs. 8.

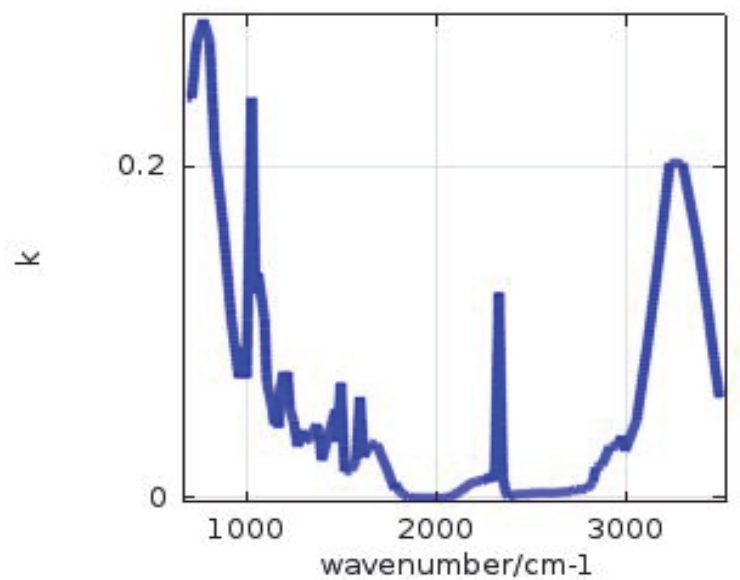

Figure 7 Calculated extinction coefficient $k_{2}$ value of contamination film from Uralane deposited on $\mathrm{Ge}$ substrate

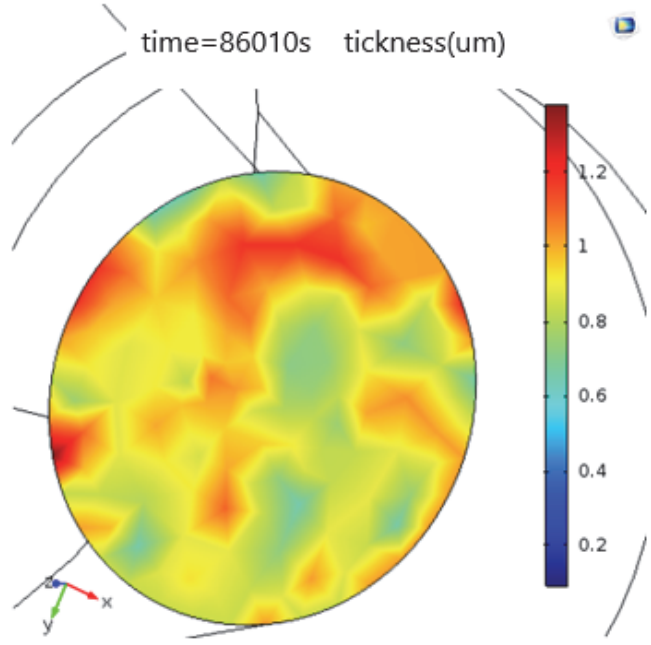

Figure 8 Calculated film thickness

\subsection{Calculation of Optical Constants of Mixing Layer and Rough Surface}

The calculation models given in section 2.3.2 were used to calculate the refractive index $n_{\mathrm{t}}$ and extinction coefficient $k_{\mathrm{t}}$ of the mixing layer using the above parameters.

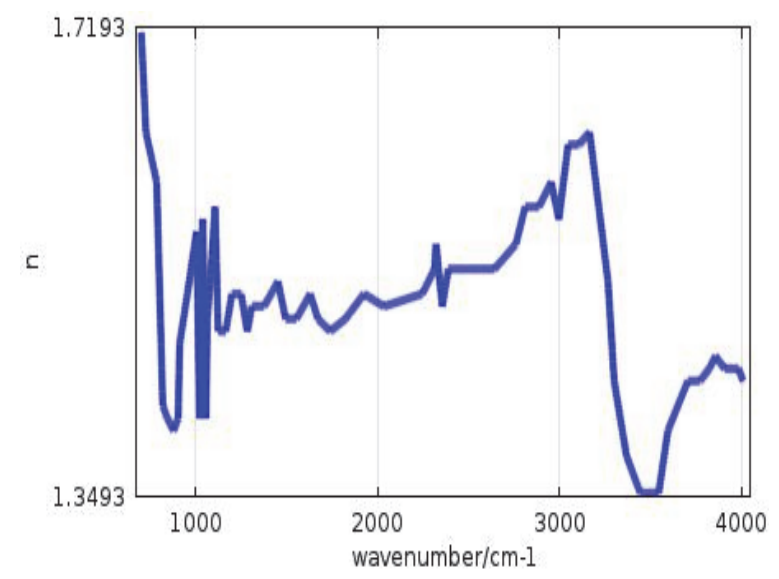

Figure 9 Calculated refractive index $n_{\mathrm{t}}$ of the mixing layer

The refractive index $n_{\text {surf }}$ and extinction coefficient $k_{\text {surf }}$ of the rough surface were calculated by replacing the first substance with air, and the thickness was calculated as $d_{\text {surf }}$ $=10$ according to Ref. [19]. The results are shown below.

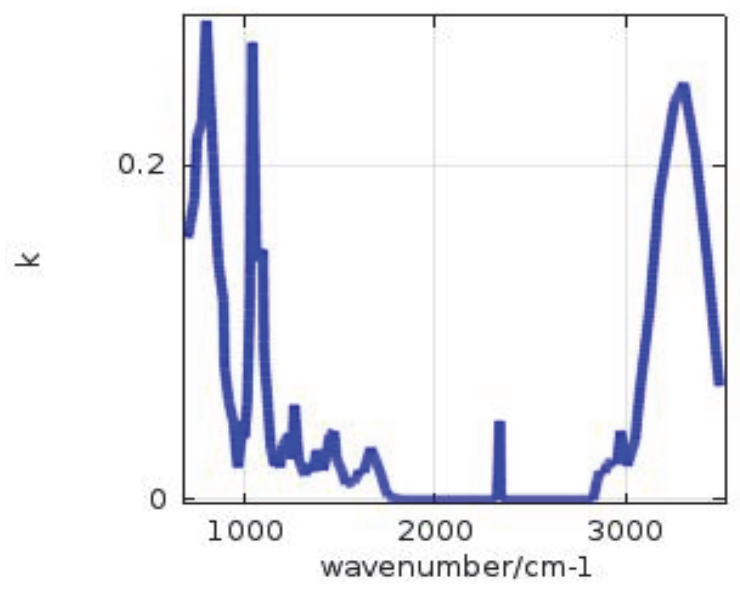

Figure 10 Calculated extinction coefficient $k_{\mathrm{t}}$ of the mixing layer 


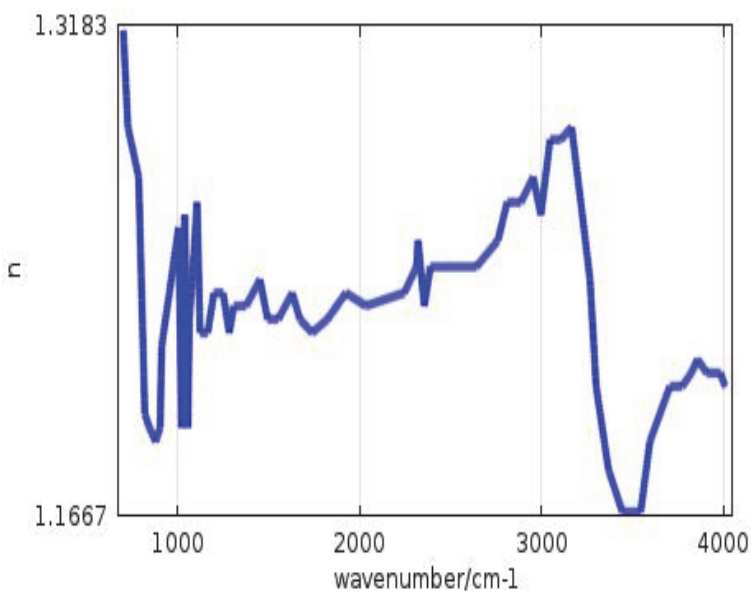

Figure 11 Calculated refractive index $n_{\text {sur }}$ of the rough surface

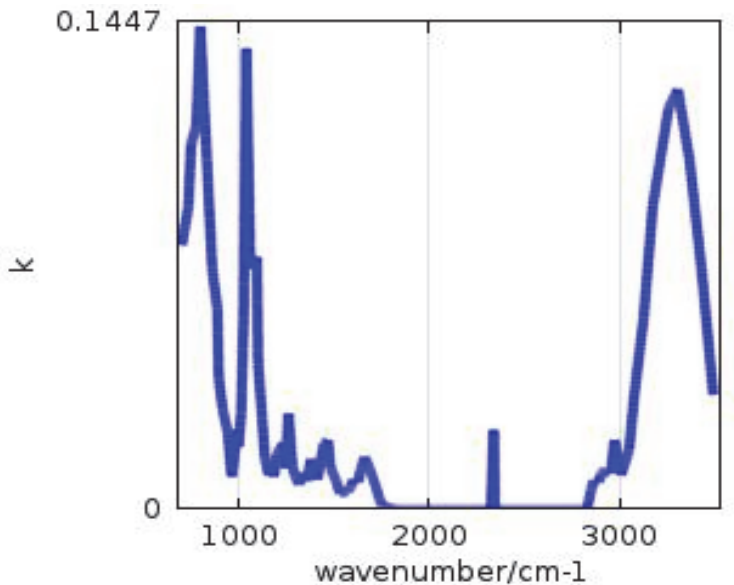

Figure 12 Calculated extinction coefficient $k_{\text {surf }}$ of the rough surface

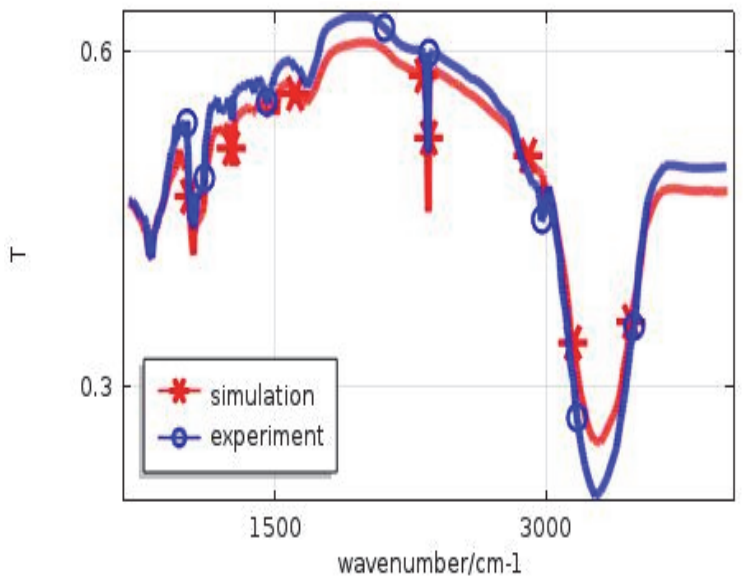

Figure 13 The calculated and measured value of the transmittance for $0.89 \mu \mathrm{m}$ thick contaminated film

\subsection{Discussion}

Comparing the calculated and measured transmittances, we find that the calculated values agree well with the measured ones. At $900-2800 \mathrm{~cm}^{-1}$ and $3500-$ $4000 \mathrm{~cm}^{-1}$, the experimental value is higher than the simulation value. However, at $3100-3500 \mathrm{~cm}^{-1}$, the measured value is lower than the calculated value. To calculate the refractive index and extinction coefficient of a mixing film, the material composition is not considered. The refractive index and extinction coefficient of different components differ, which results in the difference between the calculated and measured refractive indexes, in turn, causing the difference between the calculated and measured transmittance of the films.

Based on the obtained results, the optical contamination effect model established herein can well predict the impact of contamination on the transmittance of optical devices. Hence, it can be used to guide the anticontamination design of various space cameras and optical detectors, which is vital to ensure the long life and high reliable operation of spacecraft in orbit.

\section{CONCLUSION}

To study the effect of contamination on optical devices, Uralane and RTV silicone rubbers are used as representative materials. From the simulation and experimental result, the following conclusions are drawn:

1) The trends in the spectra for the calculated and measured transmittance of the contaminated films are consistent. However, at 900-2800 and $3500-4000 \mathrm{~cm}^{-1}$, the measured transmittance is higher than the calculated value, whereas, at $3100-3500 \mathrm{~cm}^{-1}$, the measured value becomes lower than the calculated value.

2) The discrepancy between calculated and measured results is attributed to the experimental error due to material composition.

Therefore, there is a need for further studies to measure the material composition and ratio of each component.

\section{REFERENCES}

[1] Zhao, J.-P. (2004). An introduction to the in-orbit contamination test device of the midcourse ballistic test satellite MSX. Spacecraft environment engineering, 21(2), 49-63.

[2] Tribble, A. C., Boyadjian, B., Davis, J., Haffner, J., \& McCullough, E. (1996). Contamination control engineering design guidelines for the aerospace community. NASA CR 4740.

[3] Mossman, D. L., Bostic, H. D., \& Carlos, J. R. (1987). Contamination induced degradation of optical solar reflectors in geosynchronous orbit. Proceedings SPIE, 777, 12-19. https://doi.org/10.1117/12.967062

[4] Gua, T., Hua, E.-T., \& Guoa, S. (2019). Ellipsometric study on optical properties of hydrogen plasma-treated aluminumdoped $\mathrm{ZnO}$ thin film. Vacuum, 163, 69-74. https://doi.org/10.1016/j.vacuum.2019.02.006

[5] Zhou, H., Xu, S., Zhao, Z., \& Xiang, Y. (2014). Inductively coupled hydrogen plasma processing of AZO thin films for heterojunction solar cell applications. Journal of Alloys and Compounds, 610, 107-112. https://doi.org/10.1016/j.jallcom.2014.04.216

[6] Khalid Hossain, M., Rahman, M. T., \& Basher, M. K. (2018). Influence of thickness variation of gamma-irradiated DSSC photoanodic $\mathrm{TiO}_{2}$ film on structural, morphological and optical properties. Optik (Stuttg).

[7] Basher, M. K., Khalid Hossain, M., Jalal Uddin, M., Akand, M. A. R., \& Shorowordi, K. M. (2018). Effect of the pyramidal texturization on the optical surface reflectance of monocrystalline photovoltaic silicon wafers. Optik (Stuttg).

[8] Sabzi, M. \& Mousavi Anijdan, S. H. (2018). Microstructural Analysis and Optical Properties Evaluation of Sol-Gel Heterostructured NiO-TiO 2 Film Used For Solar Panels. Ceramics International. https://doi.org/10.1016/j.ceramint.2018.10.229

[9] Paradis, P. L. et al. (2017). A 2-D transient numerical heat 
transfer model of the solar absorber plate to improve PV/T solar collector systems. Solar Energy, 153, 366-378. https://doi.org/10.1016/j.solener.2017.05.058

[10] Bertrand, W. T. \& Wood, B. E. Effects of cryogenic contaminants on optical surfaces - CALCRT thin film reflectance/transmittance model. Proceedings SPIE, 2261, 135-142.

[11] Huang, S. \& Hetrick, M. A. (1986). Preliminary Correlation of Spacecraft Contamination Flight Data with the Modified SPACE II Computer Model. AIAA/ASME 4th Thermophili's and Heat Transfer Conference, AIAA-86-1357.

[12] Khassanchine, R. H., Grigorevskiy, A. V., \& Galygin, A. N. (2004). Simulation of Outgassing Processes in Spacecraft Coatings Induced by Thermal Vacuum Influence. Journal Spacecraft Rocket, 41(3), 384-389. https://doi.org/10.2514/1.10933

[13] Frank, J. Jarossy, J. C., Pizzicaroli, E., \& Ress, B. (1980). Shuttle/payload Contamination Evaluation (SPACE) a Systems Level Contamination Model. SPIE, 216.

[14] Giunta, I., Lemcke, C., Roussel, J. F. et al. (2000). Osean/COMOVA version 1.0 contamination modelling outgassing/vent analysis tool final report.

[15] Yuan, X. X., Pang, H. W., \& Zhuou, C. L. (2007). Study on attenuation of solar cell wing power by molecular pollution. Journal of Astronautics, 28(1), 118-122.

[16] Pekker, D. \& Pekker, L. (2003 9. A method for determining the thickness and optical constants of absorbing thin films. Thin Solid Films, 425, 203-209. https://doi.org/10.1016/S0040-6090(02)01068-4

[17] Niklasson, G. A., Granqvist, C. G., \& Hunderi, O. (1981). Effective medium models for the optical properties of inhomogeneous materials. Applied Optics, 20(1), 26-30.

[18] Jin, X. H., Huang, F., Cheng, X. L. et al. (2015). Test particle Monte Carlo simulation for return flux due to ambient scatter of outgassing molecules on a circular disk. Acta Aerodynamica Sinica, 33(6), 782-798.

[19] Jiang, L., Huang, Q. S., \& Kozhevnikov, I. V. (2018). investigation of natural contamination layer growth on optical substrates. Chinese Physics C, 42(11).

[20] ASTM Designation: E 1559-03. Standard test method for contamination outgassing characteristics of spacecraft materials.

[21] Yao, R. J., Wang, Y., Wang, X. R. et al. (2007). Application of Outgassing Model of Nonmetal Materials for Satellite. Aerospace Material Technology, 33(2), 48-50.

[22] ANSI/ASTM E-595. Standard Test Method for Total Mass Loss and Collected Volatile Condensable Materials from Outgassing in a Vacuum Environment.

[23] Qiao*, J., Yang, S., Li, J., Guo, X. i Wang, Y. (2021) Dynamic Simulation of Deposition Processes of Spacecraft Molecular Contamination. Tehnički vjesnik, 28(1), 321-327. https://doi.org/10.17559/TV-20200216081552

\section{Contact information:}

\section{Jia QIAO}

(Corresponding author)

Lanzhou institute of Physics,

Lanzhou, Gansu, 730000, China

Email: qiaojia_lip@163.com 